\title{
Secretory Carrier Membrane Protein (SCAMP) deficiency influences behavior of adult flies
}

\author{
JiaLin C. Zheng ${ }^{1}$, Chook Teng Tham ${ }^{1}$, Kathleen Keatings ${ }^{1+}$, Steven Fan ${ }^{1}$, Angela Yen-Chun Liou ${ }^{1}$, \\ Yuka Numata ${ }^{1}$, Douglas Allan ${ }^{2}$ and Masayuki Numata ${ }^{1 *}$
}

1 Department of Biochemistry and Molecular Biology, University of British Columbia, Vancouver, BC, Canada

2 Department of Cellular and Physiological Sciences, University of British Columbia, Vancouver, BC, Canada

\section{Edited by:}

Maria Barile, Università degli Studi di Bari Aldo Moro, Italy

Reviewed by:

Carla Cirillo, University of Leuven, Belgium

René Massimiliano Marsano, University of Bari, Italy

*Correspondence:

Masayuki Numata, Department of Biochemistry and Molecular Biology, University of British Columbia, Vancouver, BC V6T 1Z3, Canada e-mail:mnumata@mail.ubc.ca

${ }^{\dagger}$ Present address:

Kathleen Keatings, Department of Cellular and Physiological Sciences, University of British Columbia, Vancouver, BC, Canada
Secretory Carrier Membrane Proteins (SCAMPs) are a group of tetraspanning integral membrane proteins evolutionarily conserved from insects to mammals and plants. Mammalian genomes contain five SCAMP genes SCAMP1-SCAMP5 that regulate membrane dynamics, most prominently membrane-depolarization and $\mathrm{Ca}^{2+}{ }^{2}$-induced regulated secretion, a key mechanism for neuronal and neuroendocrine signaling. However, the biological role of SCAMPs has remained poorly understood primarily owing to the lack of appropriate model organisms and behavior assays. Here we generate Drosophila Scamp null mutants and show that they exhibit reduced lifespan and behavioral abnormalities including impaired climbing, deficiency in odor associated long-term memory, and a susceptibility to heat-induced seizures. Neuron-specific restoration of Drosophila Scamp rescues all Scamp null behavioral phenotypes, indicating that the phenotypes are due to loss of neuronal Scamp. Remarkably, neuronal expression of human SCAMP genes rescues selected behavioral phenotypes of the mutants, suggesting the conserved function of SCAMPs across species. The newly developed Drosophila mutants present the first evidence that genetic depletion of SCAMP at the organismal level leads to varied behavioral abnormalities, and the obtained results indicate the importance of membrane dynamics in neuronal functions in vivo.

Keywords: Drosophila, mutant, vesicular trafficking, climbing assays, lifespan, mobility, odor-associated learning, long-term memory

\section{INTRODUCTION}

Secretory Carrier Membrane Proteins (SCAMPs) are evolutionarily conserved tetra-spanning integral membrane proteins that are broadly associated with organellar and plasma membranes (Law et al., 2012). The Drosophila genome contains a single Scamp gene on the X chromosome whereas mammals have five genes, SCAMP1-SCAMP5. Neurotransmission involves membrane-depolarization and $\mathrm{Ca}^{2+}$-dependent regulated secretion of synaptic vesicles or dense core vesicles (DCVs) targeted to pre-synaptic membrane, followed by docking and fusion in order to release neurotransmitters (Burgess and Kelly, 1987; Kim et al., 2006). Earlier biochemical studies suggested crucial roles of SCAMPs in these processes (Guo et al., 2002; Liu et al., 2005; Liao et al., 2008). Isolation of varied neuron-enriched membranebound transporters as SCAMP-binding proteins and characterization of SCAMPs' role as targeting regulators have provided additional insights. For instance, SCAMPs bind to neurotransmitter transporters (solute carrier 6, SLC6) and regulate their cell-surface targeting (Muller et al., 2006; Fjorback et al., 2011). In light of the significant role of cell-surface SLC6 in emotion and social behavior (Chen et al., 2004; Risch et al., 2009; Kristensen et al., 2011), potential impact of SCAMPs-mediated targeting of these transporters is substantial. Another potentially important SCAMP-binding partner is the neuron-enriched $\mathrm{pH}$-regulator
$\mathrm{Na}^{+} / \mathrm{H}^{+}$exchanger NHE5. NHE5 is predominantly associated with endocytic recycling organelles in resting cells (Diering et al., 2013), and SCAMPs play an important part in targeting NHE5 from endosomes to the plasma membrane in a non-neuronal heterologous expression system (Diering et al., 2009). In mature neurons, neuronal activation acutely recruits NHE5 to dendritic spines, regulates $\mathrm{pH}$ therein and greatly influences dendritic spine formation and remodeling (Diering et al., 2011). Thus, regulated targeting of NHE5 may modulate synaptic plasticity. Although the involvement of SCAMPs in activity-dependent dendritic targeting has not been tested, SCAMPs' proposed role in $\mathrm{Ca}^{2+}$ - and depolarization-induced membrane fusion and docking makes this possibility intriguing (Diering and Numata, 2014). Another class of organelle-membrane-bound $\left(\mathrm{Na}^{+}, \mathrm{K}^{+}\right) / \mathrm{H}^{+}$exchange NHE7 was also identified as a SCAMP-binding protein (Lin et al., 2005). Curiously, a multivariate association study using brain-imaging data and genome-wide single nucleotide polymorphisms identified NHE7 as a novel candidate gene for lateonset Alzheimer's disease (Meda et al., 2012). In regard to the disease-association, positional cloning has identified SCAMP5 as a candidate for autism susceptible gene (Castermans et al., 2010). Moreover, DNA microarray analysis identified SCAMP1 expression is decreased in the prefrontal cortex of schizophrenia patients (Arion et al., 2007), which may be associated with 
the symptomatic activity of this disease (Le-Niculescu et al., 2009).

The possible involvement of SCAMPs in both regulatedsecretion and membrane targeting makes these evolutionarily conserved tetra-spanning integral membrane proteins promising candidates for neuronal regulators. Despite the previous biochemical and cell biological results suggesting the fundamental significance of SCAMPs in neurons, biological roles of SCAMPs in live organisms remain untested due to the lack of suitable model organisms. We have now generated a null allele to Drosophila Scamp by imprecise excision of a P-element and report pronounced behavioral abnormalities in adults: an accelerated age-dependent decline in climbing ability, defective learning and long-term memory retention, and heat-induced seizure susceptibility.

\section{MATERIALS AND METHODS REAGENTS}

Biochemical reagents were of analytical grade or better and were obtained from Sigma-Aldrich (St Louis, MO, USA), Fisher Scientific Company (Ottawa, ON, Canada) or Bioshop Canada Inc. (Burlington, ON, Canada) unless otherwise indicated. Oligonucleotides were obtained from Invitrogen (Burlington, ON, Canada). Rabbit polyclonal antibodies against the synthetic peptide MSGSGLDENPFGEPNLDN(C-amide) corresponding to the $\mathrm{N}$-terminal 18 amino acids for Drosophila SCAMP were raised by YenZym Antibodies LLC (South San Francisco, CA, USA). Anti-myc-epitope mouse monoclonal 9E10 and rabbit polyclonal A14 antibodies that recognize EQKLISEEDL were purchased from Santa Cruz Biotechnologies, Inc. (Santa Cruz, CA, USA), anti- $\beta$-tubulin and anti-nervana antibodies were obtained from Developmental Studies Hybridoma Bank (Iowa City, IA, USA). Horseradish Peroxidase (HRP)-conjugated goat anti-mouse and anti-rabbit antibodies were purchased from Jackson Immuno Research Laboratories, Inc. (West Grove, PA, USA).

\section{DROSOPHILA STRAINS}

Strains used were: $y 1 w 67 c 23 P\{w[+m C]=G S V 1\} G S 1041 /$ Binsinscy (DGRC number 200072, Drosophila Genetic Resource Center; Kyoto, Japan), isogenized $w^{1118}$ (iso- $w^{-}$), elav-GAL4 ${ }^{C 155}$, $w^{-} ; S p / C y O ; \Delta 2-3 ; \operatorname{Sb} / T M 6( \pm \Delta 2-3)$ and $y\left[^{1}\right] w[*] P\{U A S-m C D 8::$ GFP.L $\}$ Ptp $4 E^{L L 4}$ Smox ${ }^{M B 388}$ P $\{$ neoFRT $19 A / F M 7 c$ (Stock number 44384, Bloomington Drosophila Stock Center; Bloomington, IN, USA).

\section{GENERATION OF SCAMP DEFICIENCY MUTANTS}

The P-element was mobilized from the $y 1 w 67 c 23 P\{w[+m C]=G S V 1\} G S 1041 \quad$ strain (Drosophila Genetic Resource Center) by crossing to $\Delta 2-3$ transposase. Approximately 300 mosaic-eyed progeny were crossed with the $F M 7 c$ balancer $y\left[^{1}\right] w[*] P\{U A S-m C D 8:: G F P . L\} P t p 4 E^{L L 4}$ Smox ${ }^{M B 388} P\{$ neoFRT $\} 19 A / F M 7 c$ (Bloomington Drosophila Stock Center) for two generations and the white eyed balanced progeny were PCR screened using the primers listed in Table 1. The molecular lesion generated by imprecise excision was determined by direct sequencing of genomic DNA from homozygous flies. For transgenic rescue experiments, Scamp ${ }^{63 \mathrm{~A}}$, elav-GAL $4^{c 155}$ strain was generated.

\section{GENERATION OF TRANSGENIC FLIES}

A cDNA encoding Drosophila Scamp was isolated by RT-PCR using a Drosophila embryo mRNA (Clontech, Mountain View, CA, USA) as a template. Human SCAMP1 and SCAMP5 cDNAs were previously isolated in our laboratory as described (Lin et al., 2005). In brief, first strand cDNA synthesized from human brain total RNA by the use of random primers for reverse transcription was used as a template for PCR. N-terminal myc-tag was introduced by PCR using the primers summarized in Table 2 and ligated into the pUASTattB vector (Groth et al., 2004; Fish et al., 2007; Markstein et al., 2008). The sequence of the $\mathrm{N}$-terminally myc-tagged clones was verified and

Table 1 | Primers used for Drosophila Scamp null mutant-screening.

\begin{tabular}{lll}
\hline Primer & Sequence & Genomic position \\
\hline For 1 & 5'- ACA CTA GAA TTC CAA TTG CGG AAT G-3' & $15450286-15450262$ \\
For 2 & 5'-GGT CCT AAA AAA TCA GAC CAT TGT ATA CCA G-3' & $15450091-15450061$ \\
Rev 1 & 5'-GAC GGC ATT CTA GGA ATT GTT TAT GC-3' \\
Rev 2 & 5'-CAC GTT GGC CAA CAA CGT CAT GGT G-3' & $15447149-15447174$ \\
Rev 3 & 5'-TTG TAA TCC TCA AGA GAG ACA AGG G-3' & $15447996-15448020$ \\
\hline
\end{tabular}

Table 2 | Primers used for cloning of Drosophila Scamp, human SCAMP1 and human SCAMP5.

\begin{tabular}{ll}
\hline Primer & Sequence \\
\hline Myc-DmSc For & 5'-CGG GAT CCT CGA GAA AAA ATG GAG CAG AAG CTG ATC TCC GAG GAG GAC CTG TCC GGC TCC GGT CTC GAC GAG-3' \\
DmSc Rev & 5'-GCT CTA GAG TAC CTG CTG TTG TTA AAC TGC-3' \\
Myc-hSc1 For & 5'-CGG GGT ACC AAA AAA TGG AGC AGA AGC TGA TCT CCG AGG AGG ACC TGT CGG ATT TCG ACA GTA ACC C-3' \\
hSc1 Rev & 5'-CGG GGT ACC TTA AAT CTG GTT ACC CTT GAA AGC ATT CTG-3' \\
Myc-hSc5 For & 5'-CGG ATC CCT CGA GAA AAA ATG GAG CAG AAG CTG ATC TCC GAG GAG GAC CTG GCA GAG AAA GTG AAC AAC TTC CC-3' \\
hSc5 Rev & $5^{\prime}$-GCT CTA GAT TAC ATC TCA TTG GAG TAC GTG TAA TTG GG-3'
\end{tabular}

Restriction sites were underlined and the sequence for myc-tag was italicized. 
transgenes were site specifically integrated into the attP2 genomic site by phiC31 recombinase by BestGene Inc. (Chino Hills, CA, USA).

\section{WESTERN BLOT}

Adult flies were quickly frozen in liquid nitrogen and the removed heads were lysed in RIPA buffer containing $140 \mathrm{mM} \mathrm{NaCl}, 20 \mathrm{mM}$ Tris- $\mathrm{HCl} \mathrm{pH}$ 8.0, 1\% NP40, 0.1\% SDS and 0.5\% sodium deoxycholic acid freshly supplemented with proteinase inhibitor cocktails (Roche Diagnostics, Laval, PQ, Canada). The lysate was cleared by centrifugation at 16,000 r.c.f. for $15 \mathrm{~min}$ at $4^{\circ} \mathrm{C}$ twice, and the protein concentration of the supernatant was determined with the Bradford reagent (BioRad, Hercules, CA, USA). The denatured sample was resolved in a $12 \%$ SDS-PAGE gel and transferred to a $0.45 \mu \mathrm{m}$ pore size polyvinylidene fluoride (PVDF) membrane (EMD Millipore, Billerica, MA, USA). The blot was blocked in 5\% skim milk in PBS-T $(0.075 \%$ Tween 20 in PBS $\mathrm{pH}$ 7.4) and probed with anti-Drosophila SCAMP, anti-myc, or anti-nervana antibody at room temperature for $1 \mathrm{~h}$. After extensive washing with PBS-T, the blot was incubated with HRP-conjugated secondary antibody. Following another washing in PBS-T, signal was detected with Luminata Forte Western chemilluminescent horseradish peroxidase substrate (Millipore, Billerica, MA, USA).

\section{FLY STOCK MAINTENANCE FOR BEHAVIOR EXPERIMENTS}

Homozygous Scamp deficiency mutant female flies were outcrossed with iso- $w^{-}$male, and the male Scamp hemizygous F1 progeny was tested for behavior, unless otherwise stated. Two independently-generated Scamp deficiencies were crossed and the heteroallelic Scamp deficient female F1 progeny was also tested for certain behaviors. Fly stocks were maintained at $22^{\circ} \mathrm{C}$ or $25^{\circ} \mathrm{C}$ and $70 \%$ relative humidity with $12 \mathrm{~h} / 12 \mathrm{~h}$ light/dark cycles on standard cornmeal-yeast-based food. Flies to be tested in behavior assays were collected from freshly eclosed stocks, and transferred to fresh food vials prior to the experiment. All the experiments were done in the early to midafternoon in order to avoid variation due to circadian rhythm.

\section{LIFESPAN ANALYSIS}

Null Scamp $p^{63 \mathrm{~A}}$ and precise excision Scamp $p^{211 \mathrm{~A}}$ homozygotes were collected within $16 \mathrm{~h}$ after eclosion and raised at $25^{\circ} \mathrm{C}$ in a moisture- and light-controlled environmental room. Surviving flies were counted every second day. Kaplan-Meier survival curves were generated by calculating the ratio of live flies and plotting the value as a function of incubation time.

\section{CLIMBING ASSAYS}

Climbing assays were performed as described previously (Leal and Neckameyer, 2002; Martinez et al., 2007; Perkins et al., 2010) with some modifications. Age-matched adult flies of each genotype were transferred without anesthesia to an odorless, clean polystyrene climbing-test-tube and kept in the dark for approximately $10 \mathrm{~min}$ prior to the experiment. To assay climbing, the test chamber was tapped three times to bring all flies down to the bottom; we counted the number of flies that climbed past the $7 \mathrm{~cm}$ line, from the bottom, within $8 \mathrm{~s}$. During climbing, flies were illuminated only from above to facilitate phototactic climbing. Mean numbers of flies crossing the $7 \mathrm{~cm}$ line was determined in 4 or $5 \times$ replicates per assay, and we performed 5 to 9 assays per genotype. All experiments were recorded with video for data collection.

\section{OLFACTORY CONDITIONING LEARNING AND MEMORY ASSAY}

House-made T-maze equipment and aversive odor associated learning assays were built and conducted as previously described (Tully and Quinn, 1985), with slight modifications. First, we entrained flies of all genotypes for odor-associated long-term memory. Approximately 130 post-eclosion flies (3-5 days old) of each genotype were transferred to a light-protected training chamber without anesthesia and exposed to a constant flow of odorless air (OLA) for $30 \mathrm{~min}$. Thereafter, the following entrainment procedure was followed: (i) exposure to methylcyclohexanol $(\mathrm{MCH})$ while being mildly electrically shocked with $0.5 \mathrm{~s} 60 \mathrm{~V}$ pulses (approximately $0.75 \mathrm{~mA}$ ) given at $1.5 \mathrm{~s}$ intervals for $60 \mathrm{~s}$, (ii) $45 \mathrm{~s}$ of OLA, (iii) $60 \mathrm{~s}$ of 3-octanol (OCT), and (iv) $45 \mathrm{~s}$ of OLA. After a $15 \mathrm{~min}$ spacing period, the entrainment cycle was repeated 10 times, each followed by a $15 \mathrm{~min}$ spacer. To test odorassociated long-term memory, trained flies were maintained at $22^{\circ} \mathrm{C}$ for $20 \mathrm{~h}$ before testing. Trained flies were transferred to the $\mathrm{T}$ maze apparatus without anesthesia, and exposed to $\mathrm{MCH}$ (shockassociated odor) and OCT (shock-unassociated odor) injected from opposite ends of the apparatus, but converging at the $\mathrm{T}$ maze choice point. Flies were forced to move from the choice point toward either $\mathrm{MCH}$ or OCT over a $2.5 \mathrm{~min}$ period by constant gentle agitation of the sliding center compartment at the choice point. At the end of the assay, the sliding center compartment was pulled up to separate flies on either end, and the number of flies on each side was counted. Experiments were carried out in the dark to minimize any phototaxis. Similar experiments using OCT as an associated odor and $\mathrm{MCH}$ as an non-associated odor were conducted and the learning index (LI) was calculated (Tully and Quinn, 1985). LI = (the fraction of flies avoiding the shock-associated odor) - (the fraction of flies avoiding the shock-unassociated odor). Reciprocal experiments were conducted using OCT as a shock-associated odor and $\mathrm{MCH}$ as a shock-unassociated odor, and the average value was defined as LI.

\section{HEAT-INDUCED SEIZURE ASSAYS}

Heat-induced seizure assays were carried out as described previously (Hoeffer et al., 2003) with some modifications. Newly eclosed adult flies of each genotype were grouped in triplicate in regular fly food vials (10-12 flies per vials), and were grown for 4 days at $22^{\circ} \mathrm{C}$ prior to the experiments. When the UAS-GAL4 system was used, flies were incubated at $29^{\circ} \mathrm{C}$ for 3 days followed by 1 -day incubation at $22^{\circ} \mathrm{C}$. For seizure induction, flies were transferred to fresh polystyrene fly vials and immersed in a $42.3^{\circ} \mathrm{C}$ water bath for $3 \mathrm{~min}$ at which time all the flies were paralyzed. The fly vials were then transferred to $22^{\circ} \mathrm{C}$ and the number of standing flies that recovered from the heat-shock was counted every minute for up to $30 \mathrm{~min}$. Experiments were repeated 7 times using different flies and all data were combined in the presented graphs of Kaplan-Meier failure function $[1-S(t+0)]$.

\section{STATISTICS}

Statistical analyses were conducted using Stata/IC 10.1 (StataCorp LP, College Station, TX, USA). Log-rank tests were used for 
Drosophila survival analysis and for the analysis of recovery time of heat-induced seizure assays. Means of two groups were compared with Student's $t$-tests or Wilcoxon rank-sum tests. All hypothesis tests are two-sided. Repeated measures ANOVA was used to analyze the longitudinal climbing assay data, with age as a within-subject factor, taking the proportion of climbed flies in each vial as the unit of analysis.

\section{RESULTS}

\section{GENERATION OF SCAMP NULL MUTANT FLIES}

By mobilizing the $P\{G S V 1\} G S 1041$, several imprecise excision mutants were isolated (Figure 1A). In the current study, Scamp $p^{63 \mathrm{~A}}$ was used for most of the behavioral analysis whereas Scamp $p^{50 \mathrm{~B}}$ was used to generate heterozygous $S c a m p^{63 \mathrm{~A}} / S c a m p^{50 \mathrm{~B}}$ null alleles to characterize female behaviors. As controls, precise excision revertant lines, Scamp $p^{211 \mathrm{~A}}$ and Scamp $p^{209 \mathrm{~A}}$ were used. To test protein expression in these mutants, a polyclonal antibody against the N-terminal 18 amino acids of Drosophila SCAMP was raised in rabbit. A single band approximately $35 \mathrm{kD}$ in size was detected by western blot in lysates isolated from Scamp $p^{211 \mathrm{~A}}$ and iso- $w$ (Figure 1B). Pre-incubation of the primary antibody with the epitope peptide eliminated this band. No appreciable signal was detected in Scamp $p^{63 \mathrm{~A}}$ and Scamp ${ }^{50 \mathrm{~B}}$, demonstrating the antibody's specificity. Homozygous deletion mutant flies are viable, fertile and have no apparent morphological abnormalities.

\section{SCAMP NULL ADULT FLIES HAVE SHORTENED LIFESPAN}

Decreased lifespan is frequently associated with neurodegenerative disorders in Drosophila and is often used as a "straightforward first look" at the phenotype (Lessing and Bonini, 2009). We therefore tested the survival of adult flies. The median lifespan for revertant Scamp ${ }^{211 \mathrm{~A}}$ males was 52 days whereas that of Scamp ${ }^{63 \mathrm{~A}}$ males was substantially reduced to 26 days $(-40.9 \%)(p<0.001$, log-rank test) (Figure 2). A similar reduction of life span was observed in Scamp ${ }^{63 \mathrm{~A}}$ homozygous females $(34.1 \%$ reduction compared to controls, log-rank test $p<0.001$ ).

\section{SCAMP DEFICIENT ADULT FLIES EXHIBIT SLOWER CLIMBING BEHAVIOR}

Impaired climbing behavior is frequently associated with neurological disorders, and climbing is one of the most commonly assayed behaviors because of the sensitive and robust nature of the assay that allows for testing a large number of flies (Lessing and Bonini, 2009). Homozygous Scamp deficient females $\left(S c a m p p^{63 \mathrm{~A}}\right.$ and Scamp $\left.{ }^{50 \mathrm{~B}}\right)$ and homozygous revertant Scamp $p^{211 \mathrm{~A}}$ females were crossed with isogenized $\left(\right.$ isow $\left.^{-}\right)$males, and the

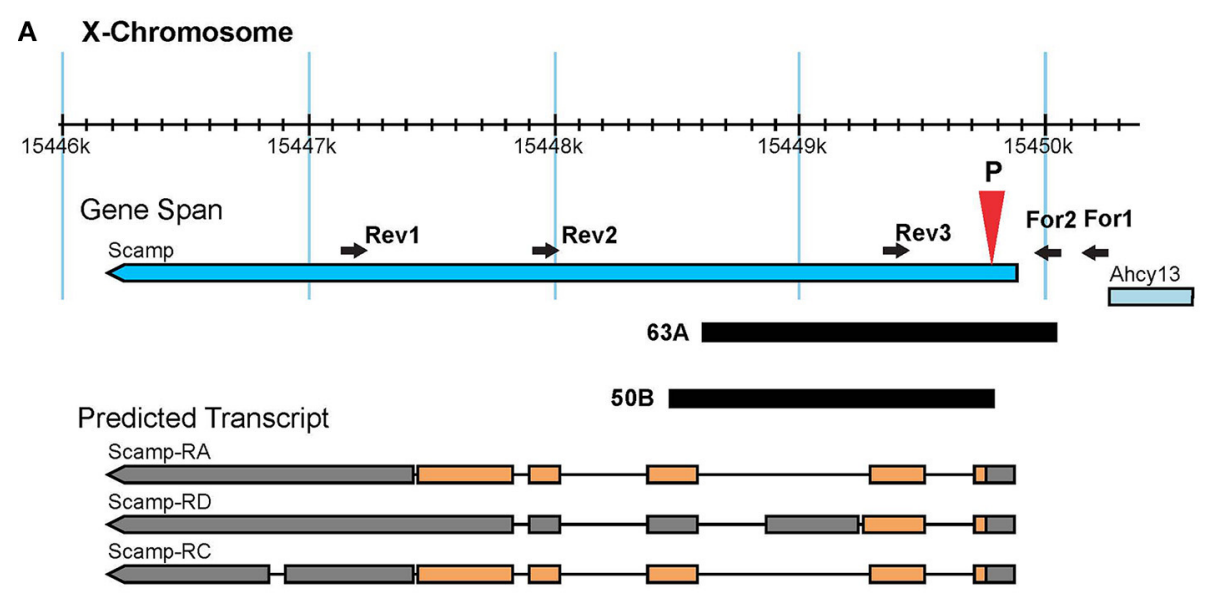

B

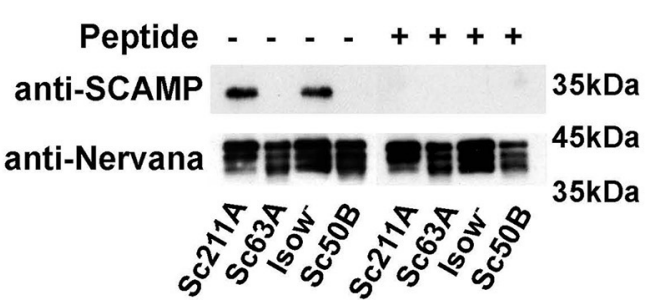

FIGURE 1 | Generation of Scamp-deficiency mutants. (A) A schematic of the genomic region of the Scamp gene and the deficiency mutants used in the study. The position of the deletion (63A and 50B) is indicated by black blocks; PCR primers (forward primers For1 and For2, and reverse primers Rev1, Rev2, and Rev3) used for screening mutants are indicated as arrowheads. P shows the position of P\{GSV1\}GS1041 insertion. Orange blocks, coding regions; Gray blocks, untranslated exons; Black lines, introns. Arrows indicate the direction of transcription. Although western blot reveals a single band with the expected size from RA and RC, the possible existence of a truncated form translated from RD cannot be completely excluded. Figure modified from Flybase (http://flybase.org/). (B) SCAMP expression in homozygous precise and imprecise excision mutants was tested by western blot. Pre-incubation of the SCAMP antibody with the epitope peptide diminished the signal to undetectable levels, assuring specificity of the antibody. The blot was probed with anti-Nervana antibody as a loading control. 

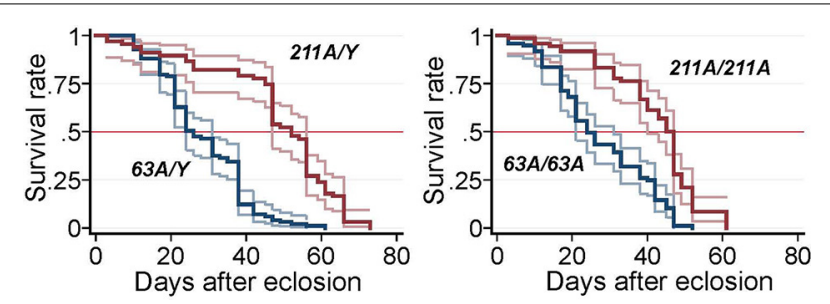

FIGURE 2 | Survival curves of Scamp mutant flies. Null males (Sc63A/Y, $N=99$ ) and precise excision controls ( $S c 211 A / Y, N=67)$, null females Sc63A/63A $(N=97)$ and controls $S c 211 A / S c 211 A(N=72)$ were raised at $25^{\circ} \mathrm{C}$ and the number of survivors was counted every other day. Pointwise $95 \%$ confidence bands of the estimated survival functions are shown by outer lines in pale colors.

F1 male nulls $\left(S c a m p^{63 \mathrm{~A}} / Y\right.$ and $\left.S c a m p^{50 \mathrm{~B}} / Y\right)$ and $S c a m p^{211 \mathrm{~A}} / Y$ controls were subjected to climbing assays 5-8 days after eclosion. Climbing of Scamp $p^{63 \mathrm{~A}} / Y$ and $S c a m p^{50 \mathrm{~B}} / Y$ was significantly slower than the Scamp $p^{211 \mathrm{~A}} / Y(p<0.001$ by Student's $t$-test, Figure 3A) where the two null mutants exhibited almost identical degrees of impairment. We next asked whether transgenic expression of Drosophila Scamp, human SCAMP1 and SCAMP5 can rescue the climbing phenotype of Drosophila Scamp nulls. When pan-neuronally expressed by elav-GAL $4^{c 155}$, all the heterologous human and Drosophila proteins were expressed similarly (Supplementary Figure 1). Transgenic expression of Drosophola Scamp under pan-neuronal promoter almost fully rescued the Scamp null climbing phenotype (Figure 3B). Either human SCAMP1 or SCAMP5 in Scamp null mutants substantially ameliorated the climbing impairment ( $p<0.05$ by Student's $t$-test), suggesting the conserved role of SCAMPs in climbing behavior. The neuronal expression of any SCAMP gene in heterozygous $S c a m p^{63 A} /+$ females did neither deteriorate nor enhance the climbing behavior, further supporting the specificity of rescue experiments (data not shown). We next examined whether female Scamp $p^{63 A} / S c a m p^{50 B}$ heteroallelic nulls at different ages also elicit retarded climbing. Scamp nulls showed significantly slower climbing than $S c a m p^{63 A} / S c a m p^{50 B}$ heteroallelic mutants at all ages except for $1-3$ days post-eclosion $(p<0.001$ or $p<0.01$ by Student $t$-tests, Figure 3C). Climbing ability of heterozygous Scamp $p^{63 A} /$ Scamp $p^{211 A}$ flies started to show only a slight decline ( $-11 \%$ on average) at 22-24 days after eclosion whereas Scamp nulls $\left(S c a m p^{63 A} / S c a m p^{50 B}\right)$ exhibited a significant decline $(-24 \%$ compared to the initial proportion) as early as 8-10 days posteclosion. Results from the repeated measures ANOVA supported that Scamp nulls perform worse and the degree of age-dependent decline significantly differs between the two strains [the interaction term of age and strain was statistically significant with $\left.F_{(4,354)}=15.85, p<0.001\right]$, indicating that Scamp deficiency accelerates age-dependent climbing impairment.

\section{SCAMP IS NECESSARY FOR OLFACTORY-ASSOCIATED MEMORY FORMATION}

A previous study showed that trans-heterozygosity for the longterm memory defective mutant CG32594 (ben) and Scamp (a P-element insertional mutant) led to a defect in long-term memory (Zhao et al., 2009). However, the effects of a Scamp null

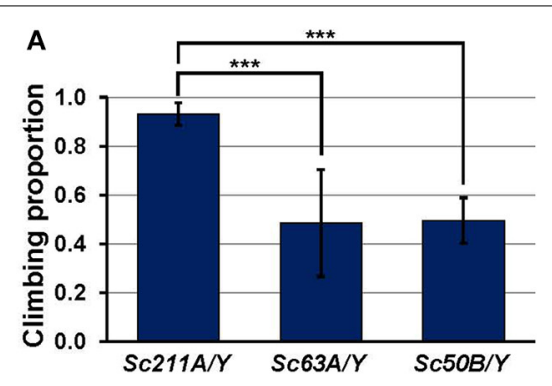

B
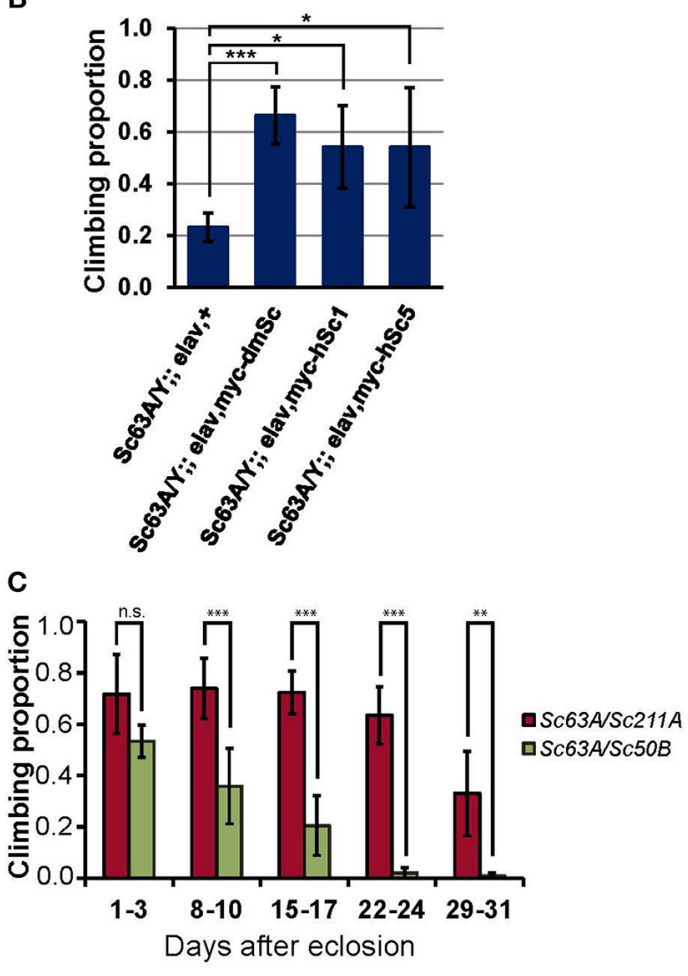

FIGURE 3 | Scamp nulls exhibit climbing impairment. (A) Outcrossed null males (Sc63A/Y and $S c 50 B / Y)$ 5-8 days post-eclosion showed significantly retarded climbing than age-matched Sc211A/Y controls. Results of climbing tests are shown as averages of $N=6, N=9, N=6$ different sets of flies for $S c 211 A / Y, S c 63 A / Y, S c 50 B / Y$, respectively. (B) Pan-neuronal expression of the myc-tagged Drosophila Scamp, human SCAMP1 or SCAMP5 rescued behavior defects of Scamp-deficiency. $N=4$ sets per genotype within 17-20 days of eclosion were assayed. (C) Heteroallelic null females (Sc63A/Sc50B) climbed markedly slower than heterozygous females ( $S c 63 A / S c 211 A)$, suggesting the recessive effect of Scamp-deficiency. The retarded climbing of nulls became apparent after 8-10 days of eclosion. $N=5$ sets per genotype (housed $N=9-14$ flies per vial) were tested weekly for 4 weeks. Mean $\pm S D$; ${ }^{* * *} p<0.001$; ${ }^{* *} p<0.01$; $* p<0.05$ by Student's $t$-test.

on long-term memory formation are unknown. To address this, we used an established aversive olfactory associated learning assay that uses electric shock for reinforcement. When exposed to 4-methylcyclohexanol $(\mathrm{MCH})$ or 3-octanol (OCT) in the Tmaze test tube under the optimized experimental condition, all genotypes evaded the odor source and moved into the opposite chamber within $2.5 \mathrm{~min}$, suggesting that Scamp mutants 
sense odors and their odor-avoidance movement is intact. Scamp null adults and age-matched revertant controls were subjected to 10 cycles of training sessions consisting of an exposure with a shock-associated- and unassociated-odors. Both genotypes reacted similarly to electric shock by freezing, falling and jumping. This memory persisted in male revertant flies after $24 \mathrm{~h}$, as they showed a strong tendency to avoid the shock-associated odor $24 \mathrm{~h}$ after training [Learning Index $(L I)=0.72 \pm 0.22$ (mean \pm SD) Figure 4A]. In contrast, Scamp nulls displayed an almost even distribution between the shock-associated and unassociated odors, showing a significant difference in LI $(L I=0.046 \pm 0.092$, $p<0.01$ by Wilcoxon rank-sum test). Similarly, heteroallelic null females exhibited significant impairment in long-term memory, as opposed to female homozygous revertants $(L I=0.015 \pm 0.11$ vs. $0.84 \pm 0.049, p<0.01$ by Wilcoxon rank-sum test, Figure $4 B$ ). These results indicate that Scamp functions in learning and longterm memory in both males and females. We next tested the effect of neuronal restoration of Drosophila Scamp, human SCAMP1 or
SCAMP5 on the long-term memory deficit of Scamp null males. Inclusion of the Drosophila UAS-Scamp $(\mathrm{dmSc})$ significantly improved the Learning Index (LI), compared to the Scamp nulls that had elav-GAL ${ }^{C 155}(L I=0.35 \pm 0.16$ vs. $-0.016 \pm 0.14, p<$ 0.01 by Wilcoxon rank-sum test, Figure $4 \mathrm{C}$ ). Interestingly, transgenic expression of human SCAMP1 $(h S c 1)$ but not SCAMP5 ( $h S c 5)$ also restored the LI of Scamp nulls albeit to a lesser extent, suggesting some conserved roles between humans SCAMP1 and Drosophila SCAMP in long-term memory formation.

\section{SCAMP NULL MUTANT FLIES EXHIBIT SUSCEPTIBILITY TO HEAT-INDUCED SEIZURES}

Elevated temperatures are a major environmental stress that influences cellular processes including enzymatic activity and the ion flux (Garrity et al., 2010), which affects membrane excitability (Peng et al., 2007) and triggers seizures (Wu et al., 1978; Hoeffer et al., 2003; Wang et al., 2004). Raising the temperature triggers seizures to flies, and the experimentally induced seizures
A

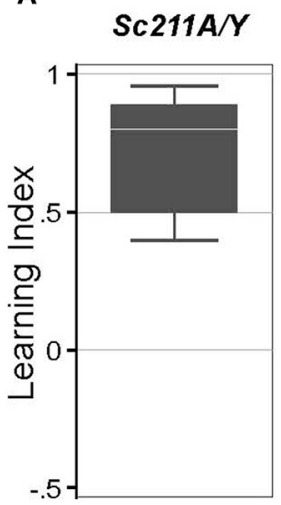

C
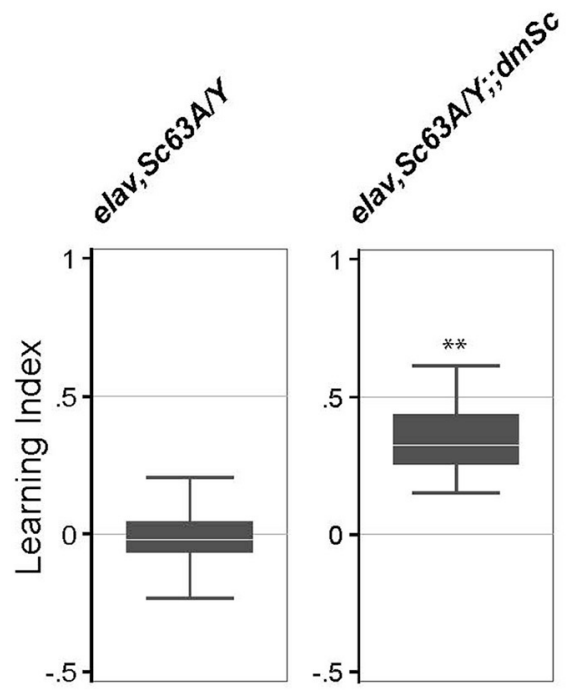

FIGURE 4 | Long-term memory is impaired in Scamp null mutants. (A) Long-term memory of the outcrossed male progeny (Sc211A/Y and Sc63A/Y) was tested $3-5$ days post-eclosion. $N=6$ experiments were conducted as shown in the box plot. (B) Long-term memory of female heterozygous Scamp nulls (Sc63A/Sc50B) and precise excision controls (Sc211A/Sc209A)
B

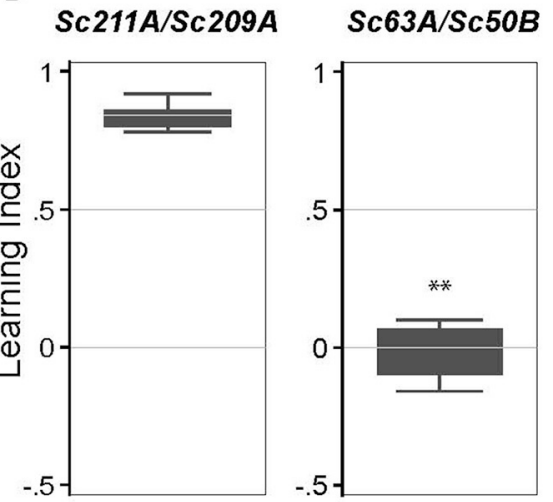

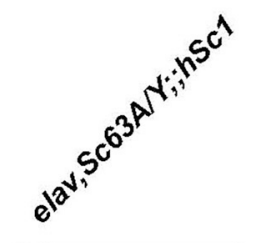
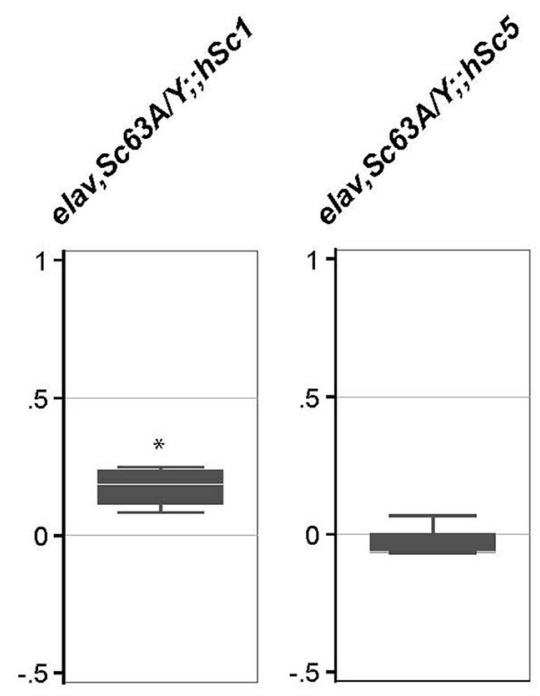

was tested $3-5$ days post-eclosion. $N=6$ for $S c 211 A / S c 209 A$ and $N=4$ for Sc63A/Sc50B. (C) elav-GAL4 and Scamp ${ }^{63 A}$ was genetically combined with UAS-myc-dmSc, myc-hSc1 or myc-hSc5 and the long-term memory of the age-matched male progeny was assessed. $N=4-6$ assessments per strain were conducted. ${ }^{* *} p<0.01 ; * p<0.05$ by Wilcoxon rank-sum test. 
was suggested to mimic some aspects of human epilepsy (Song and Tanouye, 2008). The outcrossed male progeny (Scamp ${ }^{63 \mathrm{~A}} / Y$ and $S c a m p^{211 \mathrm{~A}} / Y$ ) was subjected to heat-induced seizure susceptibility assay 5-7 days after eclosion. The median recovery time for $S c a m p^{211 A} / Y$ revertant flies was $2.5 \mathrm{~min}$ and $100 \%$ of these flies recovered within $23.5 \mathrm{~min}(N=52)$. In contrast, the median recovery time of $S c a m p p^{63 A} / Y(N=35)$ was $17.5 \mathrm{~min}$, which is considerably longer than that of revertants $(p<0.001$ by logrank test) (Figure 5A). Moreover, $28.6 \%$ of Scamp $p^{63 A} / Y$ flies did not recover even after a 30 -min incubation at $22^{\circ} \mathrm{C}(p<0.001$ by Pearson's chi ${ }^{2}$-test). Neuronal UAS-Scamp restoration experiments showed partial rescue of heat shock recovery. Median recovery time for elav-GAL4 ${ }^{C 155}, S c a m p^{63 A} / Y$ flies was $18.5 \mathrm{~min}$ $(N=81)$. Inclusion of the UAS-Scamp improved the median recovery time to $14 \mathrm{~min}(N=82)$, a small but statisticallysignificant difference ( $p<0.001$ by log-rank test) (Figure 5B). Only 75 out of $81(92.6 \%)$ elav-GAL4 ${ }^{C 155}$, Scamp $p^{63 A} / Y$ flies recovered from heat-shock within $30 \mathrm{~min}$ whereas 81 of $82(98.8 \%)$
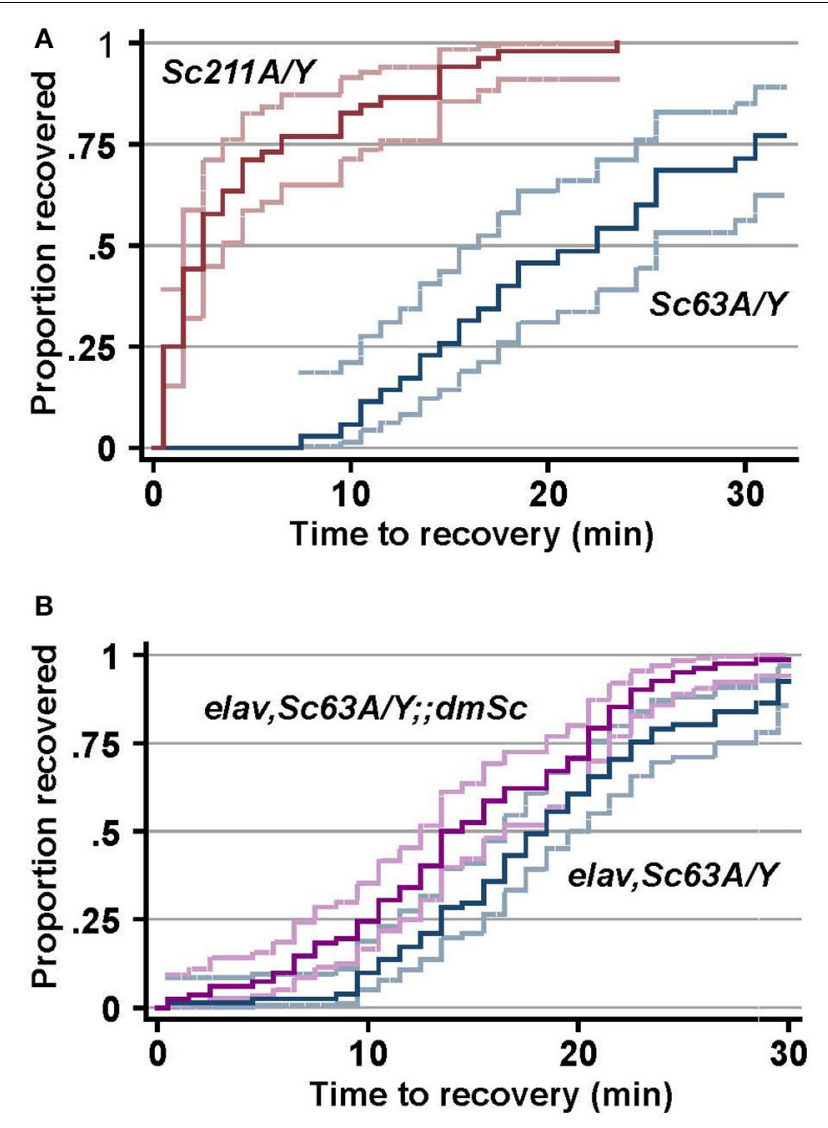

FIGURE 5 | SCAMP deficiency causes delayed recovery from heat-induced seizures. (A) The susceptibility to heat-induced seizures of the outcrossed male progeny. (B) elav-GAL4 and Scamp ${ }^{63 \mathrm{~A}}$ were genetically combined with UAS-myc-dmSc and the susceptibility to heat-induced seizures of the male progeny was determined. The log-rank test for equality of time-to-recovery functions indicated significant difference between the two strains $(p<0.001$ ). $N=81$ or 82 per genotype. Pointwise $95 \%$ confidence bands of the estimated survival function are shown by outer lines in pale colors. of the UAS-Scamp restored flies did $(p<0.05$ by Pearson's chi $^{2}$-test).

\section{DISCUSSION}

In the current study, we chose to model Scamp deficiency in Drosophila melanogaster, one of the most suitable model organisms to test stress-induced behavioral changes. We now show that Scamp deficient Drosophila mutants exhibit varied behavioral abnormalities including a decline in climbing that is exacerbated by aging, defective odor-associated long-term memory, and increased susceptibility to heat-induced seizures. Rescue of these phenotypes by neuronally-expressed Scamp demonstrate that Scamp acts within the nervous system. Moreover, restoration of these phenotypes by expression of human SCAMP1 indicates the conserved function for Scamp. Rescue of the heat-induced seizure susceptibility by the neuronal Scamp-expression was partial. Although the exact mechanism is unknown, it is possible that highly coordinated spatiotemporal Scamp-expression may be required to prevent heat-induced seizures, whereas gene expression by the UAS/GAL4 system is not finely regulated. Aging accumulates intrinsic stress that lead to deterioration in physiological processes including neuronal function. Although there are a number of other possible reasons, neurodegenerative Drosophila mutants often experience short lives (Lessing and Bonini, 2009). In this regard, it is interesting to note that Scamp nulls showed shorter lifespan than revertant controls. Although the precise mechanism remains to be determined, we postulate that the susceptibility to aging related stress, possibly via neuronal dysfunction, has caused this phenotype. Previous studies reported that SCAMP1-knockout mice were viable and fertile, and mobility of resting animals was not affected (Fernandez-Chacon et al., 1999). Furthermore, depletion of the sole SCAMP gene from the Caenorhabditis elegans genome developed no discernable effect on the sensation and spontaneous movement of the worm (Abraham et al., 2006). It is important to note that these studies assessed only movement and sensation of resting animals, and stress- or aging-induced behavioral changes in SCAMP-depleted mice, Caenorhabditis elegans or any other model organisms have never been reported. Such stress-induced behavioral changes may indeed reflect integrated brain function. The aging-dependent deterioration of climbing capability was reported to be a characteristic feature associated with Drosophila models for Parkinson's disease (Feany and Bender, 2000) and Alzheimer's disease (Iijima et al., 2004; Crowther et al., 2005). It is interesting to note that the behavioral abnormalities we identified in Scamp null flies are commonly detected in Drosophila models for human neurological disorders, including age-related neurodegenerative diseases and epilepsy.

A predicted Scamp hypomorph $w^{1118} P\{E P\} E P 1593$ (the EP transposon insertion 370 bp upstream of the transcription start site) genetically interacts with ben (CG32594) in long-term memory (Zhao et al., 2009); however, a role for Scamp in memory formation has not been tested. We now show that Scamp is necessary for odor-associated long-term memory, phenocopying the Drosophila Ben mutants. These results indicate a direct role for neuronal Scamp in learning and memory. Intriguingly, pan-neuronal expression of human SCAMP1, but not SCAMP5, 
partially rescues the long-term memory deficiency phenotype. While SCAMP1 and SCAMP5 show a high degree of similarity in their transmembrane domains, SCAMP5 lacks several putative protein-binding motifs at the N-terminal segment including AsnPro-Phe (NPF)-repeats, a Pro-rich domain and a coiled-coil domain. As a consequence, these two isoforms may modulate different biological processes. The N-terminal cytosolic extension was suggested to be crucial for proper targeting for serotonin transporters (Muller et al., 2006) and neuron-enriched $\mathrm{Na}^{+} / \mathrm{H}^{+}$exchanger NHE5 (Diering et al., 2009). It is possible that the entire structure of Drosophila SCAMP including the $\mathrm{N}$-terminal cytosolic extension, which is conserved with mammalian SCAMP1, is needed for learning and long-term memory retention. In contrast to the learning phenotype, we showed that age-dependent climbing impairment of Scamp null mutants is rescued by neuronal transgenic expression of both human SCAMP1 and SCAMP5, raising an interesting possibility that this phenotype may be regulated by a common property shared by SCAMP1 and SCAMP5. As both SCAMP1 and SCAMP5 promote $\mathrm{Ca}^{2+}$-induced cytokine secretion in rat neuroendocrine model PC12 cells (Liao et al., 2008) and immune cells (Han et al., 2009), these isoforms may exert overlapping functions in certain aspects of secretion. The potential involvement of SCAMP5 in neuronal-activity-dependent endocytosis of synaptic vesicles was also suggested (Zhao et al., 2014) while the involvement of SCAMP1 in endocytosis was also implicated (Fernandez-Chacon and Sudhof, 2000).

Identification and characterization of SCAMP-binding proteins have substantially advanced our understanding on the cellular function of SCAMPs. To better understand the biological role of SCAMPs, extending the cellular level observations to characterization of the molecular network in vivo is essential. This can be initiated by generating mutant flies lacking Scamp binding protein genes and testing their behavioral trait(s), extending to the characterization of double mutants of Scamp and its binding partners. As biologically relevant protein-protein interactions could be weak and transient, isolation of weak interactants particularly integral membrane proteins by biochemical screening methods is a challenge. Our current study has shown the utility of Drosophila to address basic questions regarding human Scamp function. Future application of the Scamp deficient mutants together with the highly sensitive behavioral assays to genome-wide screening may facilitate to unveil a novel neuronal network.

\section{CONCLUDING REMARKS}

We have established a genetically defined Drosophila model and identified quantitative phenotypes, some of which can be rescued by specific human SCAMPs. While the significance of SCAMPs in neuronal functions and their involvement in neurodegeneration have been suggested, in vivo evidence supporting the biochemical data has been missing. Our current study has bridged the existing gap in our knowledge and shown that Drosophila provides an ideal model to address basic roles of Drosophila and human SCAMPs. It will be interesting in the future to extend the genetic interaction studies testing whether mutations to the genes encoding SCAMP-binding proteins confer similar neuronal phenotypes.

\section{ACKNOWLEDGMENTS}

We thank Ian Drake and Adam Suen at LSC Equipment Services Workshop, UBC, for building the T-maze apparatus, Dr. Guy Tanentzapf (Department of Cellular and Physiological Sciences, UBC) for his suggestions on climbing assays and Dr. Michael Gordon (Department of Zoology, UBC) for discussion and comments on the manuscript. The anti-nervana monoclonal antibody developed by Paul M. Salvaterra and anti-beta-tubulin monoclonal antibody developed by Michael Klymkowsky were obtained from the Developmental Studies Hybridoma Bank, created by the NICHD of the NIH and maintained at The University of Iowa, Department of Biology, Iowa City, IA 52242. This work was supported by discovery grants from the Natural Sciences and Engineering Council of Canada (NSERC).

\section{SUPPLEMENTARY MATERIAL}

The Supplementary Material for this article can be found online at: http://www.frontiersin.org/journal/10.3389/fcell.2014. 00064/abstract

\section{Supplementary Figure 1 | Pan-neuronal transgenic expression of} myc-tagged Scamp. Either UAS-myc-dmScamp, UAS-myc-hScamp1 or UAS-myc-hScamp5 and elav-GAL4 were genetically combined with $S c a m p^{63 A}$ deficiency. Heads were separated and the equal amount of lysates isolated from each line was resolved in SDS-PAGE, transferred to a PVDF membrane and probed with either anti-myc, anti-Drosophila SCAMP $(\mathrm{dmSc})$ or anti-Nervana antibody. Nervana is used as a loading control. $\mathrm{dmSC}$ (end) = endogenous $\mathrm{dmSc}$.

\section{REFERENCES}

Abraham, C., Hutter, H., Palfreyman, M. T., Spatkowski, G., Weimer, R. M., Windoffer, R., et al. (2006). Synaptic tetraspan vesicle membrane proteins are conserved but not needed for synaptogenesis and neuronal function in Caenorhabditis elegans. Proc. Natl. Acad. Sci. U.S.A. 103, 8227-8232. doi: 10.1073/pnas.0509400103

Arion, D., Unger, T., Lewis, D. A., Levitt, P., and Mirnics, K. (2007). Molecular evidence for increased expression of genes related to immune and chaperone function in the prefrontal cortex in schizophrenia. Biol. Psychiatry 62, 711-721. doi: 10.1016/j.biopsych.2006.12.021

Burgess, T. L., and Kelly, R. B. (1987). Constitutive and regulated secretion of proteins. Annu. Rev. Cell Biol. 3, 243-293. doi: 10.1146/annurev.cb.03.110187. 001331

Castermans, D., Volders, K., Crepel, A., Backx, L., De Vos, R., Freson, K., et al. (2010). SCAMP5, NBEA and AMISYN: three candidate genes for autism involved in secretion of large dense-core vesicles. Hum. Mol. Genet. 19, 1368-1378. doi: 10.1093/hmg/ddq013

Chen, N. H., Reith, M. E., and Quick, M. W. (2004). Synaptic uptake and beyond: the sodium- and chloride-dependent neurotransmitter transporter family SLC6. Pflugers Arch. 447, 519-531. doi: 10.1007/s00424-003-1064-5

Crowther, D. C., Kinghorn, K. J., Miranda, E., Page, R., Curry, J. A., Duthie, F. A., et al. (2005). Intraneuronal Abeta, non-amyloid aggregates and neurodegeneration in a Drosophila model of Alzheimer's disease. Neuroscience 132, 123-135. doi: 10.1016/j.neuroscience.2004.12.025

Diering, G. H., Church, J., and Numata, M. (2009). Secretory carrier membrane protein 2 regulates cell-surface targeting of brain-enriched $\mathrm{Na}^{+} / \mathrm{H}^{+}$exchanger NHE5. J. Biol. Chem. 284, 13892-13903. doi: 10.1074/jbc.M807055200

Diering, G. H., Mills, F., Bamji, S. X., and Numata, M. (2011). Regulation of dendritic spine growth through activity-dependent recruitment of the brain-enriched $\mathrm{Na}^{+} / \mathrm{H}^{+}$exchanger NHE5. Mol. Biol. Cell 22, 2246-2257. doi: 10.1091/mbc.E11-01-0066

Diering, G. H., and Numata, M. (2014). Endosomal pH in neuronal signaling and synaptic transmission: role of Na/H exchanger NHE5. Front. Physiol. 4:412. doi: 10.3389/fphys.2013.00412 
Diering, G. H., Numata, Y., Fan, S., Church, J., and Numata, M. (2013). Endosomal acidification by $\mathrm{Na}^{+} / \mathrm{H}^{+}$exchanger NHE5 regulates TrkA cell-surface targeting and NGF-induced PI3K signaling. Mol. Biol. Cell 24, 3435-3448. doi: 10.1091/mbc.E12-06-0445

Feany, M. B., and Bender, W. W. (2000). A Drosophila model of Parkinson's disease. Nature 404, 394-398. doi: 10.1038/35006074

Fernandez-Chacon, R., Alvarez De Toledo, G., Hammer, R. E., and Sudhof, T. C. (1999). Analysis of SCAMP1 function in secretory vesicle exocytosis by means of gene targeting in mice. J. Biol. Chem. 274, 32551-32554. doi: 10.1074/jbc.274.46.32551

Fernandez-Chacon, R., and Sudhof, T. C. (2000). Novel SCAMPs lacking NPF repeats: ubiquitous and synaptic vesicle-specific forms implicate SCAMPs in multiple membrane-trafficking functions. J. Neurosci. 20, 7941-7950.

Fish, M. P., Groth, A. C., Calos, M. P., and Nusse, R. (2007). Creating transgenic Drosophila by microinjecting the site-specific phiC31 integrase mRNA and a transgene-containing donor plasmid. Nat. Protoc. 2, 2325-2331. doi: 10.1038/nprot.2007.328

Fjorback, A. W., Muller, H. K., Haase, J., Raarup, M. K., and Wiborg, O. (2011). Modulation of the dopamine transporter by interaction with Secretory Carrier Membrane Protein 2. Biochem. Biophys. Res. Commun. 406, 165-170. doi: 10.1016/j.bbrc.2011.01.069

Garrity, P. A., Goodman, M. B., Samuel, A. D., and Sengupta, P. (2010). Running hot and cold: behavioral strategies, neural circuits, and the molecular machinery for thermotaxis in C. elegans and Drosophila. Genes Dev. 24, 2365-2382. doi: 10.1101/gad.1953710

Groth, A. C., Fish, M., Nusse, R., and Calos, M. P. (2004). Construction of transgenic Drosophila by using the site-specific integrase from phage phiC31. Genetics 166, 1775-1782. doi: 10.1534/genetics.166.4.1775

Guo, Z., Liu, L., Cafiso, D., and Castle, D. (2002). Perturbation of a very late step of regulated exocytosis by a secretory carrier membrane protein (SCAMP2)derived peptide. J. Biol. Chem. 277, 35357-35363. doi: 10.1074/jbc.M202259200

Han, C., Chen, T., Yang, M., Li, N., Liu, H., and Cao, X. (2009). Human SCAMP5, a novel secretory carrier membrane protein, facilitates calcium-triggered cytokine secretion by interaction with SNARE machinery. J. Immunol. 182, 2986-2996. doi: 10.4049/jimmunol.0802002

Hoeffer, C. A., Sanyal, S., and Ramaswami, M. (2003). Acute induction of conserved synaptic signaling pathways in Drosophila melanogaster. J. Neurosci. 23, 6362-6372.

Iijima, K., Liu, H. P., Chiang, A. S., Hearn, S. A., Konsolaki, M., and Zhong, Y. (2004). Dissecting the pathological effects of human Abeta40 and Abeta42 in Drosophila: a potential model for Alzheimer's disease. Proc. Natl. Acad. Sci. U.S.A. 101, 6623-6628. doi: 10.1073/pnas.0400895101

Kim, T., Gondre-Lewis, M. C., Arnaoutova, I., and Loh, Y. P. (2006). Densecore secretory granule biogenesis. Physiology (Bethesda) 21, 124-133. doi: 10.1152/physiol.00043.2005

Kristensen, A. S., Andersen, J., Jorgensen, T. N., Sorensen, L., Eriksen, J., Loland, C. J., et al. (2011). SLC6 neurotransmitter transporters: structure, function, and regulation. Pharmacol. Rev. 63, 585-640. doi: 10.1124/pr.108.000869

Law, A., Chow, C.-M., and Jiang, L. (2012). Secretory carrier membrane proteins. Protoplasma 249, 269-283. doi: 10.1007/s00709-011-0295-0

Leal, S. M., and Neckameyer, W. S. (2002). Pharmacological evidence for GABAergic regulation of specific behaviors in Drosophila melanogaster. J. Neurobiol. 50, 245-261. doi: 10.1002/neu.10030

Le-Niculescu, H., Kurian, S. M., Yehyawi, N., Dike, C., Patel, S. D., Edenberg, H. J., et al. (2009). Identifying blood biomarkers for mood disorders using convergent functional genomics. Mol. Psychiatry 14, 156-174. doi: 10.1038/mp.2008.11

Lessing, D., and Bonini, N. M. (2009). Maintaining the brain: insight into human neurodegeneration from Drosophila melanogaster mutants. Nat. Rev. Genet. 10, 359-370. doi: 10.1038/nrg2563

Liao, H., Zhang, J., Shestopal, S., Szabo, G., Castle, A., and Castle, D. (2008). Nonredundant function of secretory carrier membrane protein isoforms in dense core vesicle exocytosis. Am. J. Physiol. Cell Physiol. 294, C797-C809. doi: 10.1152/ajpcell.00493.2007

Lin, P. J., Williams, W. P., Luu, Y., Molday, R. S., Orlowski, J., and Numata, M. (2005). Secretory carrier membrane proteins interact and regulate trafficking of the organellar $\left(\mathrm{Na}^{+}, \mathrm{K}^{+}\right) / \mathrm{H}^{+}$exchanger NHE7. J. Cell Sci. 118, 1885-1897. doi: 10.1242 /jcs.02315

Liu, L., Liao, H., Castle, A., Zhang, J., Casanova, J., Szabo, G., et al. (2005). SCAMP2 interacts with Arf6 and phospholipase D1 and links their function to exocytotic fusion pore formation in PC12 cells. Mol. Biol. Cell 16, 4463-4472. doi: 10.1091/mbc.E05-03-0231

Markstein, M., Pitsouli, C., Villalta, C., Celniker, S. E., and Perrimon, N. (2008). Exploiting position effects and the gypsy retrovirus insulator to engineer precisely expressed transgenes. Nat. Genet. 40, 476-483. doi: 10.1038/ ng. 101

Martinez, V. G., Javadi, C. S., Ngo, E., Ngo, L., Lagow, R. D., and Zhang, B. (2007). Age-related changes in climbing behavior and neural circuit physiology in Drosophila. Dev. Neurobiol. 67, 778-791. doi: 10.1002/dneu.20388

Meda, S. A., Narayanan, B., Liu, J., Perrone-Bizzozero, N. I., Stevens, M. C., Calhoun, V. D., et al. (2012). A large scale multivariate parallel ICA method reveals novel imaging-genetic relationships for Alzheimer's disease in the ADNI cohort. Neuroimage 60, 1608-1621. doi: 10.1016/j.neuroimage.2011. 12.076

Muller, H. K., Wiborg, O., and Haase, J. (2006). Subcellular redistribution of the serotonin transporter by secretory carrier membrane protein 2. J. Biol. Chem. 281, 28901-28909. doi: 10.1074/jbc.M602848200

Peng, I. F., Berke, B. A., Zhu, Y., Lee, W. H., Chen, W., and Wu, C. F. (2007). Temperature-dependent developmental plasticity of Drosophila neurons: cell-autonomous roles of membrane excitability, $\mathrm{Ca}^{2+}$ influx, and cAMP signaling. J. Neurosci. 27, 12611-12622. doi: 10.1523/JNEUROSCI.217907.2007

Perkins, A. D., Ellis, S. J., Asghari, P., Shamsian, A., Moore, E. D., and Tanentzapf, G. (2010). Integrin-mediated adhesion maintains sarcomeric integrity. Dev. Biol. 338, 15-27. doi: 10.1016/j.ydbio.2009.10.034

Risch, N., Herrell, R., Lehner, T., Liang, K. Y., Eaves, L., Hoh, J., et al. (2009). Interaction between the serotonin transporter gene (5-HTTLPR), stressful life events, and risk of depression: a meta-analysis. JAMA 301, 2462-2471. doi: 10.1001/jama.2009.878

Song, J., and Tanouye, M. A. (2008). From bench to drug: human seizure modeling using Drosophila. Prog. Neurobiol. 84, 182-191. doi: 10.1016/j.pneurobio.2007.10.006

Tully, T., and Quinn, W. G. (1985). Classical conditioning and retention in normal and mutant Drosophila melanogaster. J. Comp. Physiol. A 157, 263-277. doi: 10.1007/BF01350033

Wang, P., Saraswati, S., Guan, Z., Watkins, C. J., Wurtman, R. J., and Littleton, J. T. (2004). A Drosophila temperature-sensitive seizure mutant in phosphoglycerate kinase disrupts ATP generation and alters synaptic function. J. Neurosci. 24, 4518-4529. doi: 10.1523/JNEUROSCI.0542-04.2004

Wu, C. F., Ganetzky, B., Jan, L. Y., Jan, Y. N., and Benzer, S. (1978). A Drosophila mutant with a temperature-sensitive block in nerve conduction. Proc. Natl. Acad. Sci. U.S.A. 75, 4047-4051. doi: 10.1073/pnas.75.8.4047

Zhao, H., Kim, Y., Park, J., Park, D., Lee, S. E., Chang, I., et al. (2014). SCAMP5 plays a critical role in synaptic vesicle endocytosis during high neuronal activity. J. Neurosci. 34, 10085-10095. doi: 10.1523/JNEUROSCI.2156-14.2014

Zhao, H., Zheng, X., Yuan, X., Wang, L., Wang, X., Zhong, Y., et al. (2009). ben Functions with scamp during synaptic transmission and longterm memory formation in Drosophila. J. Neurosci. 29, 414-424. doi: 10.1523/JNEUROSCI.5036-07.2009

Conflict of Interest Statement: The authors declare that the research was conducted in the absence of any commercial or financial relationships that could be construed as a potential conflict of interest.

Received: 25 August 2014; accepted: 17 October 2014; published online: 18 November 2014.

Citation: Zheng JC, Tham CT, Keatings K, Fan S, Liou AY-C, Numata Y, Allan D and Numata $M$ (2014) Secretory Carrier Membrane Protein (SCAMP) deficiency influences behavior of adult flies. Front. Cell Dev. Biol. 2:64. doi: 10.3389/fcell. 2014.00064

This article was submitted to Cellular Biochemistry, a section of the journal Frontiers in Cell and Developmental Biology.

Copyright (c) 2014 Zheng, Tham, Keatings, Fan, Liou, Numata, Allan and Numata. This is an open-access article distributed under the terms of the Creative Commons Attribution License (CC BY). The use, distribution or reproduction in other forums is permitted, provided the original author(s) or licensor are credited and that the original publication in this journal is cited, in accordance with accepted academic practice. No use, distribution or reproduction is permitted which does not comply with these terms. 